\title{
Subjective Versus Objective Assessment of Cognitive Functioning in Primary Care
}

\author{
Courtney Hess, MS, Boaz Levy, PhD, Ardeshir Z. Hashmi, MD, Jacqueline Hogan, MS, \\ Sarah Greenspan, MS, Allison Elber, MA, Kathryn Falcon, MS, and \\ Daniel F. Driscoll, MD
}

Purpose: This study examined the clinical utility of highly efficient subjective and objective screens of cognitive impairment.

Method: Participants $(N=124$, age $\geq 65$, mean $=73.59$, SD $=6.26)$ completed a 2 -item questionnaire of subjective memory functioning, a brief computerized cognitive test, and the Montreal Cognitive Assessment (MoCA). Next, participants were assigned to 1 of 4 conditions, based on their subjective (low/high) and objective (impaired/unimpaired) levels of cognitive functioning. Further analysis divided the sample into age-based groups (ie, age $<75$, age $\geq 75$ ).

Results: The proportion of participants in the impaired subsample (ie, MoCA $<26$ ), who reported a high level of subjective concern about their memory, was low (ie, 0.15). Among unimpaired participants, analysis detected significant group differences across subjective memory levels $(P<.0003)$ and age $(P<.005)$ categories on one of the three tasks of the computerized test (ie, cognitive control). In contrast, the MoCA offered no differentiation between these groups.

Conclusion: Screening protocols in which cognitive testing is administered subsequent to patient complaint are prone to underdiagnosis. In addition, common dementia screens are insensitive to subjective deficits and healthy cognitive aging. Therefore, they may lead to dismissing valid concerns that deserve preventive attention. Primary care needs efficient screening tools that are sensitive to prodromal decline. (J Am Board Fam Med 2020;33:417-425.)

Keywords: Cognitive Aging, Cognitive Dysfunction, Dementia, Memory, Mental Status and Dementia Tests, Primary Health Care, Surveys and Questionnaires

\section{Introduction}

Engaging primary care in the early detection of neurocognitive disorders is likely to promote healthy aging in the community. ${ }^{1,2}$ Results of a high-quality clinical trial indicate that early behavioral and lifestyle interventions reduce the cognitive

This article was externally peer reviewed.

Submitted 28 July 2019; revised 17 December 2019; accepted 21 December 2019.

From the Department of Counseling and School Psychology, University of Massachusetts, Boston, MA (CH, BL, JH, SG, AE, KF); Cleveland Clinic, Lerner College of Medicine, Ohio (AH); Tufts University School of Medicine, Boston, MA (DD); and McLean Hospital, Belmont, MA, Harvard Medical School (BL).

Funding: Internal grant, University of MassachusettsBoston.

Conflict of interest: We hereby acknowledge that none of the authors has any conflict of interest to report. The funding source above did not play any role in conducting or designing the study, data analysis, writing the manuscript, or choosing publication venues. decline expected in at-risk aging adults. ${ }^{3}$ Additional evidence, summarized in systematic and metanalytic reviews of population-based studies, have led the Alzheimer's Association to conclude that regular physical activity, cognitive training, diet, and effective management of medical risk factors reduce the risk of cognitive impairment and dementia. ${ }^{4-6}$ Beyond notable prophylactic and clinical gains, early detection can reduce the cost of care, decrease family stress, and improve overall illness management. $^{7-10}$

These advances collectively challenge widely held notions about the limited treatability and preventability of dementia, ${ }^{11}$ or the value of making a diagnosis per se. ${ }^{12}$ In this regard, the new evidence

Corresponding author: Boaz Levy, PhD, 100 William T. Morrissey Blvd., Wheatley-02 168, Boston, MA 02125 (E-mail: boaz.levy@umb.edu). 
attenuates previous arguments against communitywide screenings. ${ }^{12,13}$ Accordingly, the debate about public screenings for dementia has now shifted in favor of implementation, as reflected by new policies, such as the Affordable Care Act, which explicitly require health assessment protocols for Medicare clients to include a cognitive evaluation during the annual wellness visit. ${ }^{14}$ This development will likely place the task of screening the public within the purview of primary care; however, the clinical protocols and guidelines for effective application in this setting have not yet been fully developed. ${ }^{15}$

At present, many primary care physicians assess cognitive functioning subsequent to patient complaints. However, this stepwise model, in which the objective assessment of cognitive functioning follows a voluntary complaint, may be insensitive to early detection of impairment. Screening all patients for subjective complaints with self-report questionnaires may improve detection rates; yet, concerns surrounding underdiagnoses are likely to persist, as some patients may be unaware of their deficits due to anosognosia, ${ }^{16,17}$ whereas others may eschew the issue altogether because of the shame and dread associated with prospective dementia. ${ }^{7}$

An alternative approach to screening may reverse the stepwise model. It would extend the objective assessment to all patients and proceed with a clinical interview subsequent to positive findings. This general strategy, however, may be fraught with psychological disadvantages. The research on motivational interviewing in primary care settings points to a clear connection between physician insight into the patient's subjective assessment of the problem (eg, awareness, significance, readiness to change) and clinical outcome, especially when interventions rely primarily on lifestyle changes. ${ }^{18,19}$ In this regard, the first conversation about the results of a medical evaluation seems to be particularly influential and should therefore be informed by the subjective assessment of patients.

Following this line of reasoning, a model of preventive screening that incorporates both subjective and objective measures, as a standard of care, may address the aforementioned concerns; yet, it still needs to contend with clinical decisions around disagreement between objective test results and subjective patient report. Specifically, it is unclear when a negative (ie, unimpaired) outcome of an objective screening procedure would be sufficient to assuage patient concerns about cognitive dysfunction, and thereby avoid a costly referral to neuro-cognitive diagnostics. This issue raises questions related to screen sensitivity, particularly in prodromal patients who report a worrisome decline in cognitive functioning, while maintaining adequate functional adjustment for their circumstances.

To address these questions, the current study aimed to assess the agreement between subjective and objective measures in a community sample. The main hypothesis was that sensitivity to subjective complaints would be associated with test challenge. More specifically, common dementia screens (eg, MiniCog, Mini Mental Status Examination [MMSE], Montreal Cognitive Assessment [MoCA]) suffer from a ceiling effect, because they are not designed to assess cognitive limits. Instead, they indicate impairment by failing a lower, predetermined set of challenges. For this reason, they may mask prodromal declines and, as a result, contradict valid complaints. In theory, the low ceiling of ordinary dementia screens decreases their sensitivity to the prodromal decline that occurs while patients still function within the range of normal limits.

A higher ceiling provides the performance range needed to differentiate prodromal decline from normal aging. Thus, in otherwise comparative samples, on a test with a higher ceiling and more challenging tasks, prodromal patients would probably score lower, on average, than cognitively healthy people. Stated differently, the intersection between lower test performance and subjective concerns may elevate the probability for prodromal decline. ${ }^{17}$ This hypothesis carries implications to practice. Alongside the outcome of standard dementia screening, indication from a more challenging test would probably help to interpret patients' complaints more accurately and, consequently, lead to better decisions about subsequent care. Of note, although prodromal status is difficult to establish with a high degree of certainty, either in research or in practice, the combination of subjective deficits and a lower-thanexpected performance on objective measures may point to increased risk that deserves attention.

\section{Method \\ Sample}

One-hundred and twenty-one participants (age mean $=73.59, \mathrm{SD}=6.26$, range, 65 to 91 ) were 
included in the current study. Participants were selected from a larger pool of data $(n=206)$ that were collected as a part of another project. ${ }^{15}$ The participants in the current sample were selected based on their age $(\geq 65)$ and MoCA score $(\geq 21$, ie, excluding scores below the range of mild cognitive impairment). Approximately one-third of participants were referred to the study from 2 primary care offices in Boston, Massachusetts, and the rest of the sample was recruited from the community at large. Aside from age and MoCA performance, inclusion criteria for participation required sufficient English reading proficiency for understanding the test instructions and signing a written informed consent. Exclusion criteria included visual impairments or acute medical conditions that may affect cognitive functioning (eg, delirium, high fever, intense physical pain, recent brain trauma). Demographic data for the current study sample are shown in Table 1. The study was approved by the Beth Israel Deaconess Medical Center institutional review board via expedited review.

\section{Measures}

\section{Montreal Cognitive Assessment}

The MoCA is a prolifically researched and widely implemented screening tool for detecting mild cognitive impairment..$^{20-22}$ It is clinician administered and takes approximately 15 minutes to complete. The MoCA is scored on a 30-point scale, with a clinical cutoff score of $<26 .{ }^{21}$ The MoCA testing materials used in the current study were accessed through an authorized account registered on mocatest.org.

\section{The Computerized Test}

The third objective cognitive test employed in the current study consisted of a brief self-administered computerized task that included 3 parts: (1) balloon popping (BP) to assess fine motor speed by popping 16 balloons on the screen; (2) number sequencing (NS) to assess visual processing speed and sequential attention by clicking on 16 nonconsecutive numbers on the screen in ascending order, placed in the same locations as the balloons; and (3) evenodd switching (EOS) to assess cognitive/executive control by shifting back and forth between 16 even and odd nonconsecutive numbers in ascending order. In the present study, test performance was measured by completion time, with faster execution indicating better cognitive performance. The
Table 1. Participant Demographics

\begin{tabular}{lc}
\hline Demographic Variable & $\mathrm{N}(\%)$ \\
\hline Sex & \\
Female & $82(67.8)$ \\
Male & $39(32.2)$ \\
Race & \\
Asians or Asian Americans & $1(0.8)$ \\
Black or African Americans & $1(0.8)$ \\
White & $119(98.4)$ \\
Education & \\
High school diploma & $14(11.6)$ \\
Associate's degree & $16(13.2)$ \\
Bachelor's degree & $49(40.5)$ \\
Master's degree & $25(20.7)$ \\
Doctoral degree & $17(14.0)$ \\
Marital status & \\
Single & $15(12.4)$ \\
Married & $73(60.3)$ \\
Divorced & $11(9.10)$ \\
Widowed & $22(18.2)$ \\
Employment status & $102(84.3)$ \\
Retired & $10(8.3)$ \\
Employed full time & $6(5.0)$ \\
Employed part time & $2(1.7)$ \\
Volunteering & $1(0.8) \mathrm{a}$ \\
Long-term disability & \\
\hline &
\end{tabular}

theoretical considerations for the test design have been seen in previous conceptual work ${ }^{23}$ and a study reporting its convergence validity with the MoCA through machine learning methods. ${ }^{15} \mathrm{~A}$ demonstration of the test is available online and can be accessed with Google Chrome or Firefox browsers via http://sequencingtest.demo.umb.edu.

\section{Subjective Memory Assessment}

To assess subjective memory perception, participants completed a 2-item questionnaire. For each item, participants rated level of concern on a 4point Likert-type scale, with scores ranging from 0 to 3. Participants were asked to evaluate their memory functioning at present time (ie, "How would you rate your memory overall?”; Poor $=0$, Fair $=1$, Good $=2$, Excellent $=3$ ) and compare it to 10 years ago (ie, "How would you rate your memory compared with 10 years ago?"; Much worse $=0$, Worse $=1$, A bit weaker $=2$, About the same $=3$ ). In the current sample, internal consistency of the subjective memory assessment was in the acceptable range $\alpha=0.723,95 \%$ CI, 0.629-0.817. 


\section{Procedure}

After confirmation of eligibility for the study and signing the informed consent, participants completed the MoCA, which was administered by trained research assistants, in addition to 2 other measures not analyzed in the current study (ie, Mini Mental State Examination and the Patient Health Questionnaire- $4^{15}$ ). Next, participants filled out demographic questions and the subjective memory questionnaire. The session concluded after participants completed the self-administered computerized cognitive test.

\section{Data Analysis and Study Design}

Participants were assigned to high and low levels of subjective concern. High subjective concern was determined by a total score $\leq 2$, generally indicating ratings on the lower end of the 4-point Likert-type scale on both items (ie, indicating that current memory functioning is either "poor" or "fair" in general and "worse" or "much worse" in comparison to 10 years ago). Next, participants were assigned into 1 of 4 quadrants in a $2 \times 2$ contingency table, according to their subjective (ie, low/ high level of subjective memory concerns) and objective (ie, impaired/unimpaired status, based on MoCA clinical cutoff score, $<26$ ) classifications.

The study also examined an alternative approach to classification, in which objective level of cognitive functioning was determined by the computerized test instead of MoCA (see Table 2). In this procedure, participants were assigned to higher and lower levels of objective cognitive functioning by kmeans clustering of the test completion times (ie, NS and EOS). A Yuen test for trimmed means was employed to examine the difference in MoCA scores between the groups.

To evaluate the clinical utility of the subjectiveobjective stepwise model, analysis examined the rate of high-level subjective concerns within the subset of participants who were classified as impaired by objective measures. Confidence intervals for this estimate were based on the binomial probability function.

To examine test sensitivity to subjective complaints and cognitive aging in unimpaired participants, a Yuen test ${ }^{24}$ for trimmed means compared objective cognitive test performances across high and low levels of subjective concerns as well as across age groups (ie, age $<75$ vs age $\geq 75$ ). Effect size was examined with an alternative measure to
Table 2. Sample Classification Based on Objective and Subjective Cognitive Measures

\begin{tabular}{lccr}
\hline & \multicolumn{3}{c}{ Subjective Measure } \\
\cline { 2 - 4 } Objective Measures & Low & High & Total \\
\hline MoCA clinical cutoff score & & & \\
Unimpaired $(\geq 26)$ & 79 & 22 & 101 \\
Impaired $(<26)$ & 17 & 3 & 20 \\
$\quad$ Total & 96 & 25 & 121 \\
Computerized tests (k-means clustering) & & \\
Unimpaired & 81 & 16 & 97 \\
Impaired & 15 & 9 & 24 \\
$\quad$ Total & 96 & 25 & 121 \\
\hline
\end{tabular}

MoCA, Montreal Cognitive Assessment.

Cohen's $d{ }^{25}$ which allows for heteroscedasticity in unequal samples. Interpretation of its numeric value was calibrated to $0.5 \mathrm{Xd}$ (ie, small $=0.1$, medium $=$ 0.25 , large $=0.4$ ) to adjust for discrepant standard errors. ${ }^{26}$ Bonferroni correction for multiple comparisons was applied to maintain study-wise type 1 error $<0.05$.

\section{Results}

\section{Descriptive Statistics}

In the entire sample, the mean(SD) and median MoCA scores were 27.30(2.18) and 28.0 (minimum $=21$, maximum $=30$ ), respectively. Approximately $79 \%$ of participants $(n=101)$ scored above the clinical cutoff score of the MoCA, and about $21 \%$ of scores $(n=12)$ were within the mild cognitive impairment range. In the subsample of unimpaired participants, based on MoCA $\geq 26$, the mean (SD) and median scores of MoCA were 28.05(1.35) and 28, respectively. When the subsample of unimpaired participants was determined by the computerized test $(n=97)$, the mean(SD) and median scores of MoCA were almost identical: 27.62(1.85) and 28 , respectively. These analyses suggest that the sample was relatively high functioning.

With respect to subjective memory, on the item that assesses current functioning, the high concern and low concern groups had a mean(SD) of 0.80 (0.40) and 1.94(0.55), respectively. Qualitatively, the mean ratings of the group with higher concern fall between the "poor" and "fair" response options, whereas the mean of the group with lower concern is associated with a "good" rating. On the quantitative side, analysis indicated a substantial difference 
between the groups, evidenced by an effect size of $2.27(t=9.53, \mathrm{SE}=0.12, P<.000)$.

Analysis of the second questionnaire item, which required participants to rate their memory relative to 10 years ago, revealed a similar pattern. The mean rating of the groups with higher concerns was slightly lower than "worse" (mean $=0.92, \mathrm{SD}=$ 0.50 ), whereas the corresponding mean rating in the group with lower concerns was between the "a bit weaker" and "about the same" response options (mean $=2.25, \mathrm{SD}=0.81 ; t=12.20, \mathrm{SE}=0.11$, effect size $=2.74, P<.000)$. Overall, the qualitative and numeric (ie, effect size) differences in ratings between the groups are consistent with the study design.

Measurement of completion time for the computerized test was of interest in the current study, in light of the procedural efficiency warranted by the practical constraints of the primary care setting. The mean(SD) completion time for BP, NS, and EOS was 25.78(12.54), 44.17(20.14), and 93.33 (79.17) seconds, respectively.

\section{Evaluation of Clinical Models}

As indicated by Table 2, in the impaired subsample, the proportion of participants who reported high levels of subjective concern was 0.15 (ie, 3/20). Based on the binomial probability function, the CI for this estimate is $0.03-0.37$. When the classification criteria for impairment was based on the computerized test, the proportion estimate increased to 0.375 (CI, 0.20-0.59). These findings suggest that, whereas the computerized test seems to be more sensitive to subjective deficits than the MoCA, objective testing subsequent to patient complaint is largely prone to underdiagnosis of cognitive impairment.

Further analysis evaluated the sensitivity of MoCA and the computerized test to subjective concerns and age in unimpaired participants (MoCA $\geq$ 26). Figure 1 displays the box plots that compare test performance across the high and low levels of subjective concerns. The Yuen test results for these comparisons are summarized in Table 3. As the table indicates, this analysis detected significant group differences only for the EOS test. This result remained significant when the samples were matched for age (mean age difference $=1.51$, $\mathrm{SE}=1.35, P<.26)$ by excluding participants older than 85 years old $(\mathrm{n} 1=18, \mathrm{n} 2=79$, tmean $1=106.27$, tmean $2=72.73, d f=12.3, \quad \mathrm{SE}=12.5, \quad$ Yuen test statistic $=2.68, P<.01)$. Of note, the difference in MoCA score between the groups was smaller than 1 test point. Figure 2 displays the box plots that compare performance across age groups on the MoCA and EOS. Consistent with these box plots, Table 3 reveals a significant group difference only in EOS performance. BP, NS, and MoCA did not approach significance in these comparisons. Thus, EOS was the only test that demonstrated sensitivity to both age and subjective level of concern in unimpaired participants.

\section{Discussion}

The current study evaluated the subjective-objective stepwise model for assessing cognitive functioning in primary care. Results suggest that a protocol in which objective testing is contingent on patient complaint or subjective deficits is inadequate for detecting impairment. Most participants who scored in the impaired range on testing reported a relatively low level of subjective concerns. Consistent with previous findings, ${ }^{27}$ this outcome indicates that proactive cognitive screening is essential for detecting impairment. Stated differently, clinical protocols that rely on patient complaints, or subjective report, will likely result in substantial underdiagnosis. Assessment of cognitive functioning in primary care should therefore apply both subjective and objective measures in tandem, within a preventative model.

The study further revealed that alleviating subjective concerns with objective test results can sometimes dismiss valid complaints, even when the assessment is based on measures recommended by best practices, such as the MoCA. ${ }^{22}$ In this study, the MoCA was unable to distinguish between unimpaired participants with high and low subjective concerns. It was also insensitive to cognitive aging at large in a relatively high-functioning sample. In contrast, the same analysis detected significant group differences for the EOS task, consistent with the direction of subjective concerns and the age of participants.

The fact that analysis failed to detect significant group differences for BP and NS may be attributed to low power on a statistical level; at the same time, based on effect size estimates, these tests clearly offered weaker group differentiation than the EOS. Although these tasks are not bounded by a ceiling (ie, given that performance is based on 
Figure 1. Test sensitivity to subjective concerns in unimpaired participants (MoCA $\geq 26)$. Abbreviations: BP, balloon popping (assessing motor speed); EOS, even-odd switching (assessing cognitive control/flexibility in set shifting); MoCA, Montreal Cognitive Assessment; NS, number sequencing (assessing processing speed).

b. BP performance across levels of subjective concern

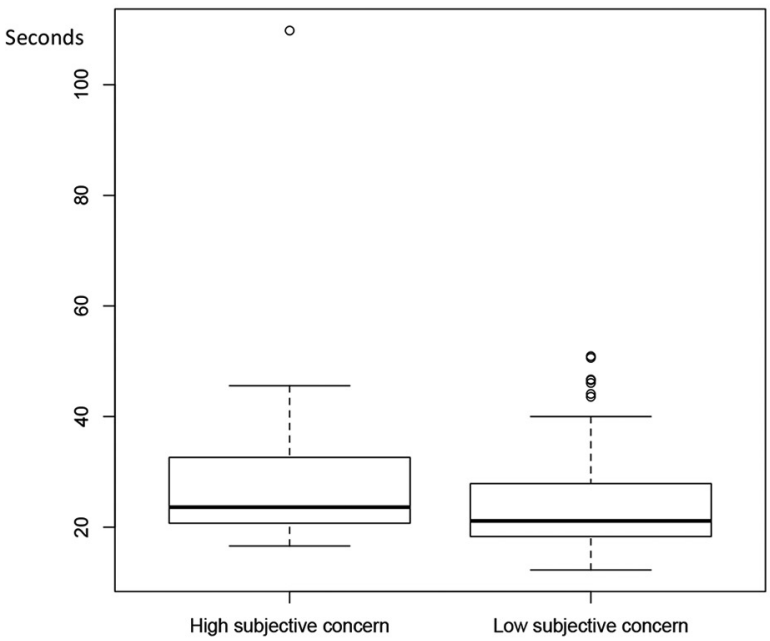

d. EOS performance across levels of subjective concern

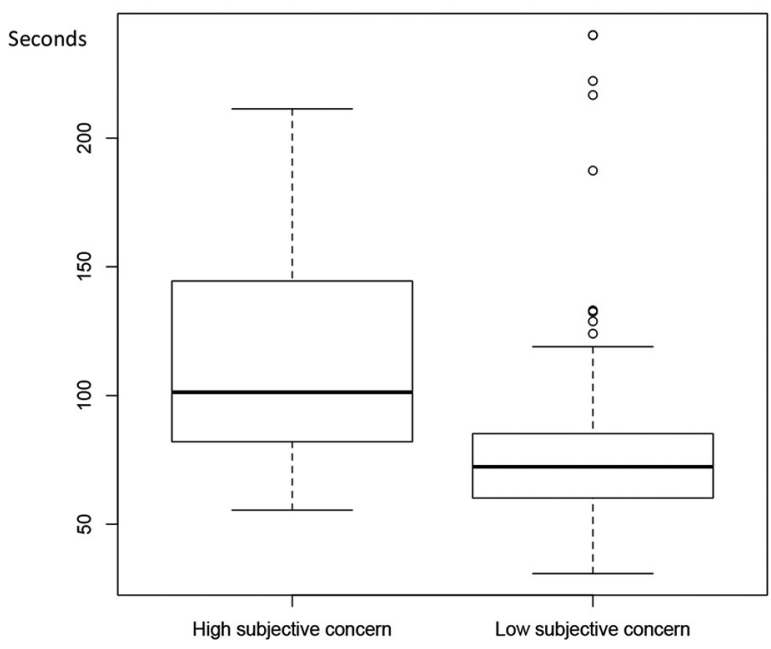

a. NS performance across levels of subjective concern

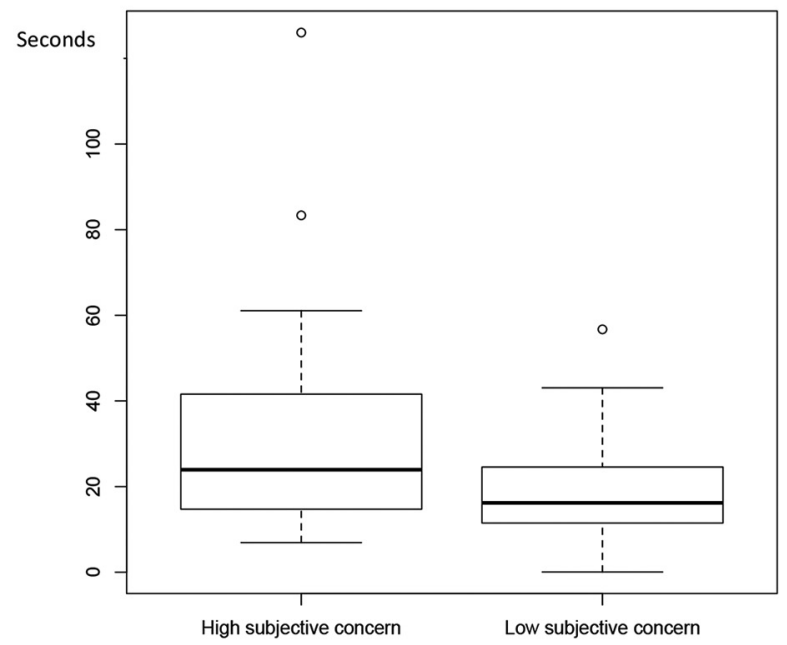

c. MoCA performance across levels of subjective concern

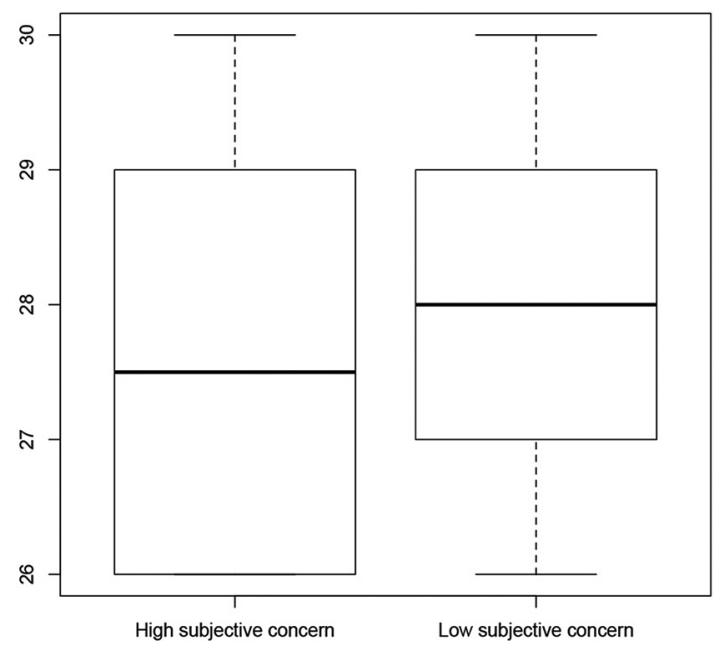

completion time), they do present a substantially easier challenge than EOS to participants. Thus, test sensitivity within the unimpaired range is enhanced by the combination of elevated ceiling and higher challenge.

Moreover, the association that emerged between subjective concerns and EOS in unimpaired participants carries clinical implications. It largely indicates that subjective concerns can map on to an objective reality that is difficult to discern with ordinary dementia screens or behavioral observations. In this respect, the sharp decline in functional demands that typically follows retirement can mask a subjectively palpable deterioration. This study did not use a longitudinal design; yet, its results are consistent with the hypothesis that the subjective appraisal of abnormal cognitive decline may, in some cases, reflect prodromal progression. ${ }^{28}$ In other words, even when patients score within normal limits on dementia screens, their subjective sense of precipitous decline may be accurate. ${ }^{29,30}$ They may notice, and report to their physician, a worrisome rate of cognitive deterioration in daily living before conspicuous functional deficits arise. $^{31,32}$ 
Table 3. Yuen Comparisons of Cognitive Tests across Subjective Levels of Concern and Age Groups

\begin{tabular}{lllrrrrrrr}
\hline Groups Compared & Test & $\mathrm{n} 1, \mathrm{n} 2$ & tmean1 & tmean2 & SE & Yuen & $d f$ & $P$ Value & E.size \\
\hline High versus low levels of subjective & BP & 22,79 & 24.71 & 21.90 & 2.13 & 1.31 & 17.95 & 0.20 & 0.12 \\
memory concern & NS & 22,79 & 45.22 & 37.95 & 5.49 & 1.32 & 14.03 & 0.20 & 0.12 \\
& EOS & 22,79 & 107.97 & 72.73 & 10.34 & 3.40 & 14.83 & $0.0003^{* * *}$ & 0.32 \\
& MoCA & 22,79 & 27.64 & 28.1 & 0.45 & 1.18 & 16.65 & 0.25 & 0.11 \\
Age, based on $<75, \geq 75$ split & EOS & 32,69 & 100.04 & 71.17 & 9.4 & 3.06 & 23.04 & $0.005^{* * *}$ & 0.29 \\
& MoCA & 32,69 & 28.00 & 28.11 & 0.37 & 0.30 & 29.59 & 0.76 & 0.02 \\
\hline
\end{tabular}

BP, balloon popping; EOS, even-odd sequencing; E.size, effect size; MoCA, Montreal Cognitive Assessment; NS, number sequencing; tmean, trimmed mean; Yuen, test statistic, SE, standard error; $d f$, degrees of freedom.

The magnitude of group differences in EOS completion times deserves particular attention. High level of subjective concerns was associated with $48 \%$ longer completion times. Similarly, older participants completed the task at a pace that is $35 \%$ slower than their younger counterparts. The EOS task requires participants to keep track of 2 series of numbers simultaneously by switching their attention, back and forth, between them continuously. This type of set shifting is demanding and required by many aspects of higher functioning. Difficulty keeping track of information and shifting attention quickly decreases the number of tasks people can complete in a single day. In addition, it limits the ability to engage with complexity, especially under time pressure. Therefore, patients who are worried about their level of functioning at home or work and, at the same time, perform on the EOS significantly slower than expected during an office visit may be at higher risk for deterioration than others. In other words, even when impairment does not emerge on a dementia screen, subjective concerns may deserve medical attention, particularly when they are coupled with a relatively lower performance on a more challenging test.

Several limitations of the study deserve mention. First, the sample did not include minority groups, and most participants had a relatively high level of education. Research has already established that education and culture are important factors to consider for proper clinical interpretation of cognitive test results. ${ }^{33}$ These factors may equally affect subjective reports of cognitive functioning, although the research on this topic is more limited. Second, the subjective memory instrument, albeit useful for the present study, is not yet sufficiently

Figure 2. Test sensitivity to healthy aging (MoCA $\geq 26$ ). Abbreviations: EOS, even-odd switching (assessing cognitive control/flexibility in set shifting); MoCA, Montreal Cognitive Assessment.

a. EOS performance across age groups

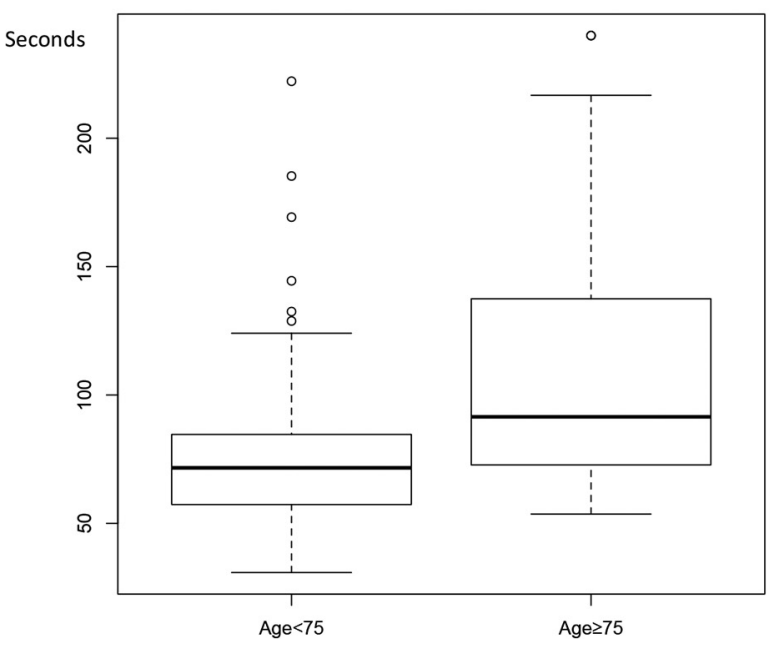

b. MoCA scores across age groups

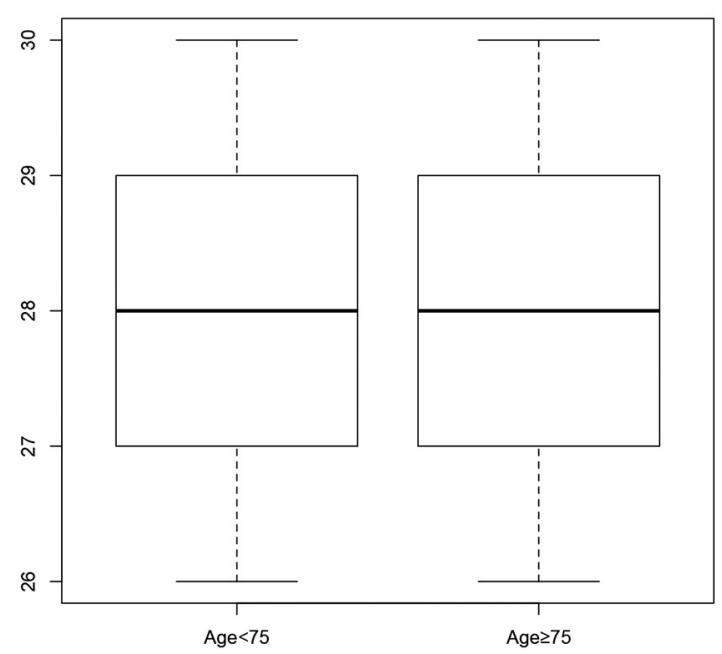


developed for clinical use. Third, the questionnaire used the term "memory" to capture subjective evaluation of mental faculties more generally. A more inclusive term like "cognitive functioning" may have been more technically accurate yet potentially less familiar to many people. Of note, the MoCA assesses memory as a specific cognitive domain; however, it was less sensitive to subjective memory concerns than the computerized test, which technically assesses executive functioning. Thus, the effect of the semantic inaccuracy, while unknown, may have been relatively small. Finally, the small sample size of impaired participants ( $\mathrm{n}=20$ by MoCA criteria and $n=24$ by the computerized test criteria) may have affected the estimate of the proportions of subjective-objective congruence in this group (ie, 0.15 and 0.375 , respectively). However, the overall conclusion that relying on subjective reports for impairment is largely unsatisfactory seems largely consistent with previous investigations. ${ }^{34,35}$

Despite these limitations, the results of this study suggest that a cognitive assessment in primary care should incorporate both objective and subjective measures. The study further clarifies the limitations of ordinary dementia screens in addressing subjective memory complaints, as well as the need to use efficient tests that are sensitive to subtle yet deleterious changes in normal cognitive functioning. In this respect, the optimal test would be sensitive to accelerated cognitive losses that occur before development of mild cognitive impairment. From a practical perspective, tests like the EOS are particularly appealing for the primary care setting, because they are self-administered and take less than 2 minutes to complete.

Future studies may expand this work to validate procedures, instruments, and protocols against diagnostic outcome. This process will inevitably increase the expertise of primary care in cognitive evaluations and patient access to care.

To see this article online, please go to: http://jabfm.org/content/ 33/3/417.full.

\section{References}

1. Clionsky M, Clionsky E. Dementia screening: saying no to the USPSTF and yes to brief cognitive evaluation. J Alzheimers Dis Parkinsonism 2014;04:e132.

2. Grober E, Mowrey WB, Ehrlich AR, et al. Twostage screening for early dementia in primary care. J Clin Exp Neuropsychol 2016;38:1038-49.
3. Ngandu T, Lehtisalo J, Kivipelto M, et al. A 2-year multidomain intervention of diet, exercise, cognitive training, and vascular risk monitoring versus control to prevent cognitive decline in at-risk elderly people (FINGER): a randomized controlled trial. Lancet 2015;385:2255-63.

4. Andrieu S, Coley N, Lovestone S, Aisen PS, Vellas B. Prevention of sporadic Alzheimer's disease: lessons learned from clinical trials and future directions. Lancet Neurology 2015;14:926-44.

5. Baumgart M, Snyder HM, Carrillo MC, Fazio S, Kim H, Johns H. Summary of the evidence on modifiable risk factors for cognitive decline and dementia: a population-based perspective. Alzheimers Dement 2015;11:718-26.

6. Peters R, Booth A, Rockwood K, Peters J, D'Este C, Anstey KJ. Combining modifiable risk factors and risk of dementia: a systematic review and metaanalysis. BMJ Open 2019;9:e022846.

7. Dubois B, Padovani A, Scheltens P, Rossi A, Dell'Agnello G. Timely diagnosis for Alzheimer's disease: a literature review on benefits and challenges. JAD 2015;49:617-31.

8. Getsios D, Blume S, Ishak KJ, Maclaine G, Hernández L. An economic evaluation of early assessment for Alzheimer's disease in the United Kingdom. Alzheimers Dement 2012;8:22-30.

9. Leifer BP. Early diagnosis of Alzheimer's disease: clinical and economic benefits. J Am Geriatr Soc 2003;51:S281-S288.

10. Weimer DL, Sager MA. Early identification and treatment of Alzheimer's disease: social and fiscal outcomes. Alzheimers Dement 2009;5:215-26.

11. Daviglus ML, Bell CC, Berrettini W. National Institutes of Health state-of-the-science conference statement: preventing Alzheimer disease and cognitive decline. Ann Intern Med 2010;153:176-81.

12. Portet F, Ousset PJ, Visser PJ. Mild cognitive impairment (MCI) in medical practice: a critical review of the concept and new diagnostic procedure. Report of the MCI Working Group of the European Consortium on Alzheimer's Disease. J Neurol Neurosurg Psychiatry 2006;77:714-8.

13. Moyer VA. Screening for cognitive impairment in older adults: US Preventive Services Task Force recommendation statement. Ann Intern Med 2014;160:791-7.

14. Cordell CB, Medicare Detection of Cognitive Impairment Workgroup, Borson S, Boustani M, et al. Alzheimer's Association recommendations for operationalizing the detection of cognitive impairment during the Medicare annual wellness visit in a primary care setting. Alzheimers Dement 2013;9:141-50.

15. Levy B, Hess C, Hogan J, et al. Machine learning enhances the efficiency of cognitive screenings for primary care. J Geriatr Psychiatry Neurol 2019;32: 137-44. 
16. Morris RG, Mograbi DC. Anosognosia, autobiographical memory and self-knowledge in Alzheimer's disease. Cortex 2013;49:1553-65.

17. Sunderaraman $\mathrm{P}$, Cosentino S. Integrating the constructs of anosognosia and metacognition: a review of recent findings in dementia. Curr Neurol Neurosci Rep 2017;17:27.

18. Anstiss T. Motivational interview in primary care. J Clin Psychol Med Settings 2009;16:87-93.

19. Rollnick S, Miller WR, Butler CC. Motivational interviewing in health care: helping patients change behavior. New York: Guilford Press; 2008.

20. Lee JY, Lee DW, Cho SJ. Brief screening for mild cognitive impairment in elderly outpatient clinic: validation of the Korean version of the Montreal Cognitive Assessment. Int J Geriatr Psychiatry 2008;21:104-10.

21. Nasreddine ZS, Phillips NA, Bédirian V, et al. The Montreal Cognitive Assessment, MoCA: a brief screening tool for mild cognitive impairment. J Am Geriatr Soc 2005;53:695-9.

22. Smith T, Gildeh N, Holmes C. The Montreal Cognitive Assessment: validity and utility in a memory clinic setting. Can J Psychiatry 2007;52: 329-32.

23. Levy B, Tsoy E, Gable S. Developing cognitive markers of Alzheimer's disease for primary care: implications for behavioral and global prevention. JAD 2016;54:1259-72.

24. Yuen KK. The two-sample trimmed $t$ for unequal population variances. Biometrica 1974;61:165-70.

25. Kulinskaya E, Staudte RG. Interval estimates of weighted effect sizes in the one-way heteroscedastic ANOVA. Br J Math Stat Psychol 2006;59: 97-111.
26. Kulinskaya E, Morgenthaler S, Staudte RG. Metaanalysis: a guide to calibrating and combining statistical evidence. New York: Wiley; 2008.

27. Borson S, Frank L, Bayley PJ, et al. Improving dementia care: the role of screening and detection of cognitive impairment. Alzheimers Dement 2013;9:151-9.

28. Sheehan B. Assessment scales in dementia. Ther Adv Neurol Disord 2012;5:349-58.

29. Buckley RF, Maruff P, Ames D, et al. Subjective memory decline predicts greater rates of clinical progression in preclinical Alzheimer's disease. Alzheimers Dement 2016;12:796-804.

30. Garcia-Ptacek S, Eriksdotter M, Jelic V, PortaEtessam J, Kåreholt I, Manzano Palomo S. Subjective cognitive impairment: towards early identification of Alzheimer's disease. Neurología 2016;31:562-71.

31. Rönnlund M, Sundström A, Adolfsson R, Nilsson LG. Subjective memory impairment in older adults predicts future dementia independent of baseline memory performance: evidence from the Betula prospective cohort study. Alzheimers Dement 2015;11:1385-92.

32. Jorm AF, Christensen H, Korten AE, Jacomb PA, Henderson AS. Memory complaints as a precursor of memory impairment in older people: a longitudinal analysis over 7-8 years. Psychol Med 2001;31:441-9.

33. Brickman AM, Cabo R, Manly JJ. Ethical issues in cross-cultural neuropsychology. Appl Neuropsychol 2006;13:91-100.

34. Starkstein SE. Anosognosia in Alzheimer's disease: diagnosis, frequency, mechanism and clinical correlates. Cortex 2014;61:64-73.

35. Leicht H, Berwig M, Gertz HJ. Anosognosia in Alzheimer's disease: the roles of impairment levels in assessment of insight across domains. J Int Neuropsychol Soc 2010;16:463-73. 